PACS: 72.40.+w, 72.20.j, 84.60.j.

\title{
Investigation of the effect of external factors (heating, exposition to radiation) on the electrophysical parameters of barrier heterostructures $\boldsymbol{p}$-AlGaAs-p-GaAs-n-GaAs
}

\author{
N.L. Dmitruk ${ }^{1}$, D.M. Yodgorova ${ }^{2}$, A.V. Karimov ${ }^{2}$, R.V. Konakova ${ }^{1}$, Ya.Ya. Kudryk ${ }^{1}$, and A.V. Sachenko ${ }^{1}$ \\ ${ }^{1}$ Institute of Semiconductor Physics, NAS of Ukraine, 41, prospect Nauky, 03028 Kyiv, Ukraine \\ E-mail:dmitruk@isp.kiev.ua \\ 2 Physical-Technical Institute of the Scientific Association "Physics-Sun" \\ of the Academy of Sciences of Republic of Uzbekistan, Tashkent, Uzbekistan
}

\begin{abstract}
Over a wide temperature range $(77 \ldots 400 \mathrm{~K})$, we studied $I-V$ curves of photoelectric converter (solar cell) prototypes made on the basis of $p-\mathrm{Al}_{\mathrm{x}} \mathrm{Ga}_{1-\mathrm{x}} \mathrm{As}-$ $p$-GaAs- $n$-GaAs $-n^{+}$-GaAs heteroepitaxial structures grown on smooth or microrelief $n^{+}$-GaAs substrates using the standard liquid phase epitaxy (LPE) and the capillary LPE from a confined volume, as well as the effect of some external factors (thermal treatment and ${ }^{60} \mathrm{Co} \gamma$-irradiation) on the current flow mechanisms. At temperatures $\leq 200 \mathrm{~K}$, the tunnel component of the forward current was predominant in all the smooth samples studied up to the voltages close to $1 \mathrm{~V}$, while at room temperatures all three components (diffusion, recombination and tunnel) were of the same order. This is evidenced, in particular, by the dependencys of the effective ideality factor on the applied voltage. Predominance of the tunnel current component in a wide temperature range at small biases was observed for all the solar cells obtained on the textured $n^{+}$-GaAs substrates. In this case, an additional factor favoring the increase of the tunnel current component was relief irregularities with small curvature radii.
\end{abstract}

Keywords: photoelectric converter, liquid phase epitaxy (LPE), microrelief, capillary LPE, $I-V$ curves, tunnel current component, solar cell, thermal treatment, $\gamma$-irradiation.

Manuscript received 24.12.04; accepted for publication 18.05.05.

\section{Introduction}

Texturing the front surface is traditionally used in photoconverters of solar energy into electric one (solar cells, (SC)) to reduce optical losses (by reflection) and increase the SC efficiency. Contrary to the above, the object of this work is development and investigation of such GaAs-based SC where textured surface not only serves as a passive element (improving the use of light due to the reduction of losses by reflection) but immediately realizes photoconversion being an active interface in a hetero- or $p-n$ junction $[1,2]$. This places additional constraints on structure of such interface, since its departure from planarity may distort the electric fields of potential barrier and increase the electron losses by recombination. That is why the use of textured surface (interface) as a SC active element involves provision of its structural perfection.

Furthermore, the open-circuit voltage $V_{\mathrm{OC}}$ in $\mathrm{SC}$ based on $p$-AlGaAs- $p$-GaAs- $n$-GaAs heterosystem essentially depends on the dominant current flow mechanism. For small lifetimes (diffusion lengths) of minority charge carriers in the $p$ - and $n$-regions of $\mathrm{GaAs}$, the dominant component is usually that related to recombination in the space-charge region (SCR) of $p-n$ junction. And in structures obtained by molecular epitaxy with the photoelectric conversion efficiency of about 22 to $24 \%$, the recombination current in quasineutral bulk (i.e., diffusion current) prevails [3].

On the other hand, in $[4,5]$, it was shown that, in $p-n$ junctions on the basis of III-V semiconductors with sufficiently high density of growth dislocations crossing $p-n$ junction, the recombination current component related to tunneling through dislocations excess charge carriers may appear along with those mentioned above.

Here, we present the results of our investigations of dark $I-V$ curves taken for SC prototypes based on $p$-AlGaAs- - -GaAs- $n$-GaAs heterostructures obtained by LPE (both the standard and capillary techniques), as well as the effect of some external factors (thermal treatment and ${ }^{60} \mathrm{Co} \gamma$-irradiation) on the current flow mechanisms. First the current flow mechanism in the initial heterostructures of both types (made with standard and capillary LPE techniques) will be considered. 

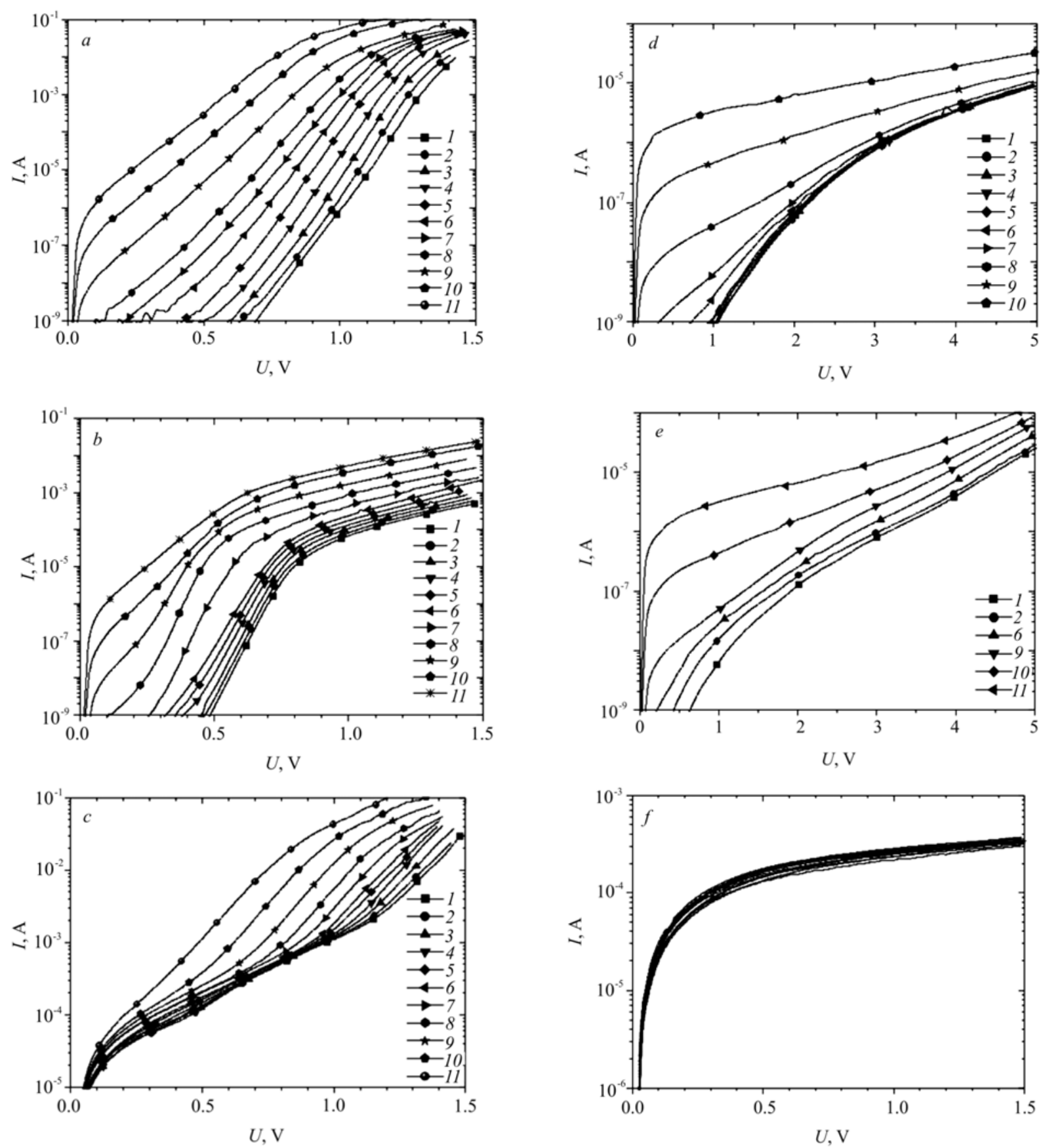

Fig. 1. Dark forward (a, b, c) and reverse (d, e, f) branches of SC $I-V$ curves taken at $T=77 \mathrm{~K}$ (1), 100 (2), 125 (3), 150 (4), 175 (5) 200 (6), 225 (7), 250 (8), 300 (9), 350 (10) and 400 (11); a, b, d, e - for the standard LPE and c, f - for the capillary LPE.

\section{Experimental results and discussion}

2.1. The mechanisms of recombination current in SC made on the basis of morphologically nonuniform AlGaAs-GaAs heterostructures

Shown in Fig. 1 are dark $I-V$ curves taken for $\mathrm{SC}$ with in 77 to $400 \mathrm{~K}$ temperature range.
By comparing the above $I-V$ curves, one can see that, in the samples made by the capillary LPE technique, the tunnel current component is dominant practically over the whole temperature range, both in forward $(V<0.5 \mathrm{~V})$ and reverse directions. And in the samples obtained by the standard LPE, the considerable tunnel current component is observed (for both forward and reverse branches of $I-V$ curves) in 77 to $200 \mathrm{~K}$ temperature range only. This fact is evidenced, in particular, by presence of portions with a big ideality

(C) 2005, V. Lashkaryov Institute of Semiconductor Physics, National Academy of Sciences of Ukraine 


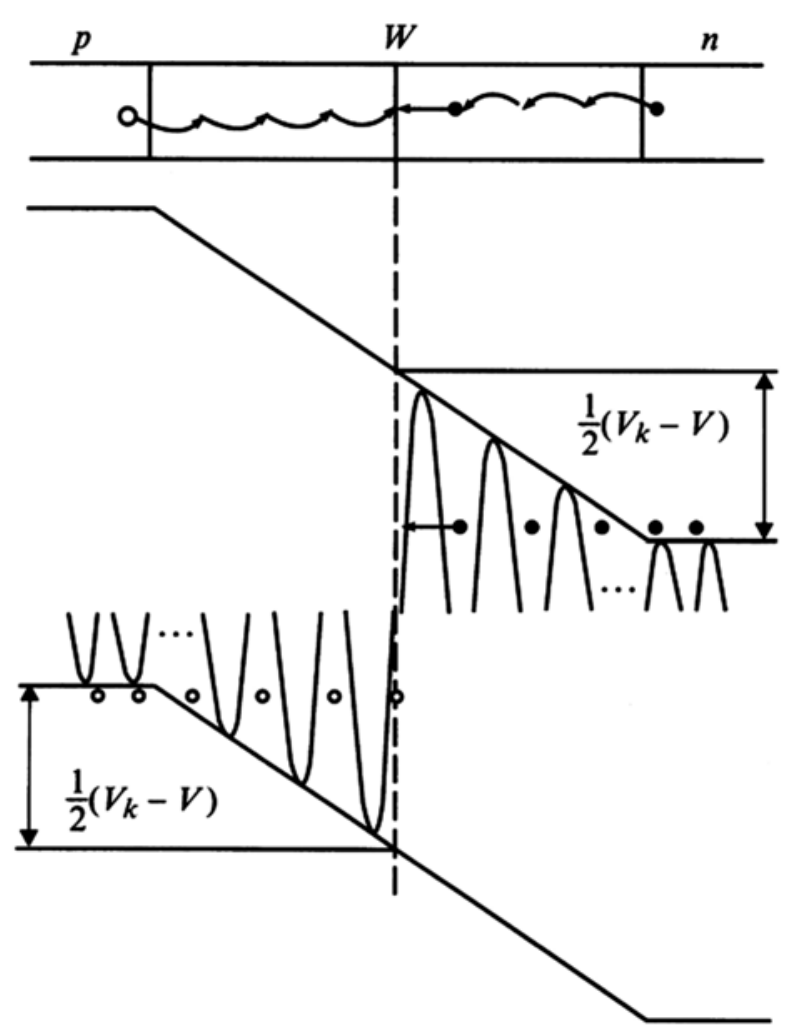

Fig. 2. Dislocation shunting model (multiple tunneling through dislocation), as applied to $p$ - $n$ junction (straight arrow shows the limiting tunneling transition).

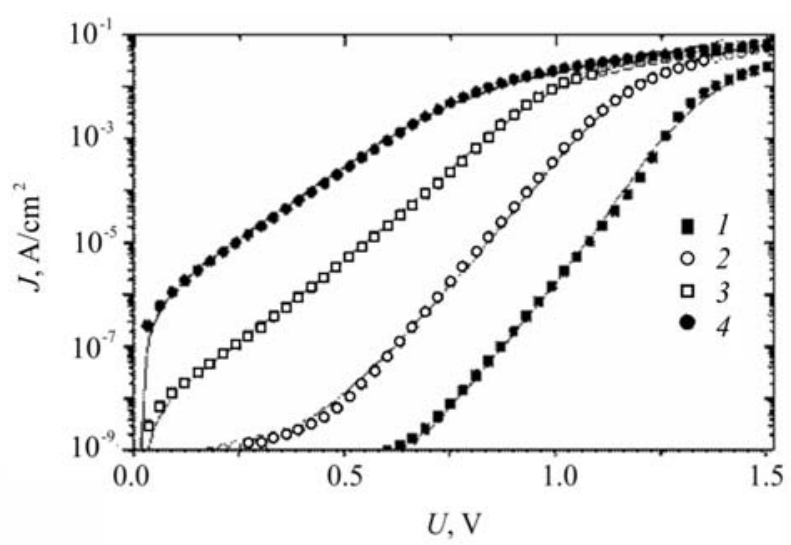

Fig. 3. The experimental and (coordinated with them) calculated forward $I-V$ curves for the SC sample made by the standard LPE technique, taken at $T=100$ (1), 200 (2), 300 (3) and $400 \mathrm{~K}(4)$.

factor $n$ in the forward branches of $I-V$ curves at low temperatures, while the reverse branches have portions with the power current dependency on voltage, $J \sim V^{m} \cdot(m \approx 4 \div 5)[4-6]$.

Theoretical treatment of $I-V$ curves taken at forward biases was performed for the $\mathrm{SC}$ equivalent circuit with commonly used parameters. A three-exponential model was applied, with allowance made for the diffusion, recombination, and tunnel currents. The ideality factors for the diffusion and recombination components of the forward current were taken equal to 1 and 2 .
Taking into account the results of [5] for the case when the diode equivalent circuit with lumped parameters is valid, one can write down the diode total forward current density $J(V, T)$ as

$$
\begin{aligned}
& J(V, T)=J_{0 D}(T) \exp \left(\frac{q\left(V-J(V, T) R_{S}\right)}{k T}\right)+ \\
& +J_{0 R}(T) \exp \left(\frac{q\left(V-J(V, T) R_{S}\right)}{2 k T}\right)+ \\
& +J_{0 T}(T) \exp \left(\frac{q\left(V-J(V, T) R_{S}\right)}{\varepsilon_{T}}\right)+ \\
& +\frac{V-J(V, T) R_{S}}{R_{S H}} .
\end{aligned}
$$

Here $q$ is the electron charge; $J_{0 D}(T) \sim n_{i}^{2}(T)$ and $J_{0 R}(T) \sim n_{i}(T)$ are is the diffusion and recombination saturation current densities, respectively; $n_{i}(T)=\left(N_{c}(T) N_{v}(T) \exp \left(-E_{g}(T) / k T\right)\right)^{1 / 2}$ is the charge carrier concentration in the intrinsic semiconductor; $N_{c}(T) \cdot\left(N_{v}(T)\right)$ is the effective density of states in the conduction (valence) band; $J_{0 T}(T)$ is the tunnel saturation current density (slightly depends on temperature); $\quad \varepsilon_{T} \sim \sqrt{r / m *}$ is the characteristic tunneling energy; $m^{*}$ is the effective mass; $r$ is the curvature radius of the top of parabolic barrier that limits tunneling; $R_{S}\left(R_{S H}\right)$ is the series (shunt) resistance.

Shown in Fig. 2 is an energy diagram of a symmetric nondegenerate $p-n$ junction (see $[4,5]$ ) illustrating multiple tunneling through dislocations. The paths of electrons and holes tunneling through the chains of deep impurities localized at dislocations are shown. The barriers between the potential wells near the localized centers are approximated with parabolas.

We managed to fit the experimental and calculated $I-V$ curves. To do that, however, we had to assume in some cases that the series resistance can grow as the temperature goes down. This is possible if the fluctuation model for the current flow is realized in heavily doped $p$-AlGaAs and $p$-GaAs regions at low temperatures.

In addition, in some experimental samples either sufficiently low (about $10^{4} \mathrm{Ohm}$ ) shunt resistances $R_{S H}$ or high (about $10^{2} \mathrm{Ohm}$ ) series resistances $R_{S}$ were realized, depending on the SC manufacturing technology.

Shown in Fig. 3 are the experimental and (coordinated with them) calculated $I-V$ curves for a $\mathrm{SC}$ sample made by the standard LPE technique. We obtained $R_{S H} \approx 10^{7} \mathrm{Ohm}$ and $R_{S} \approx 5 \mathrm{Ohm}$. In this sample, the tunnel component of the forward current prevailed up to $V \approx 1 \mathrm{~V}$ at $T \leq 200 \mathrm{~K}$. At the room temperature, all three components of the forward current were of the same order of magnitude. This was evidenced, in particular, by the ideality factor $n$ dependencies on the 


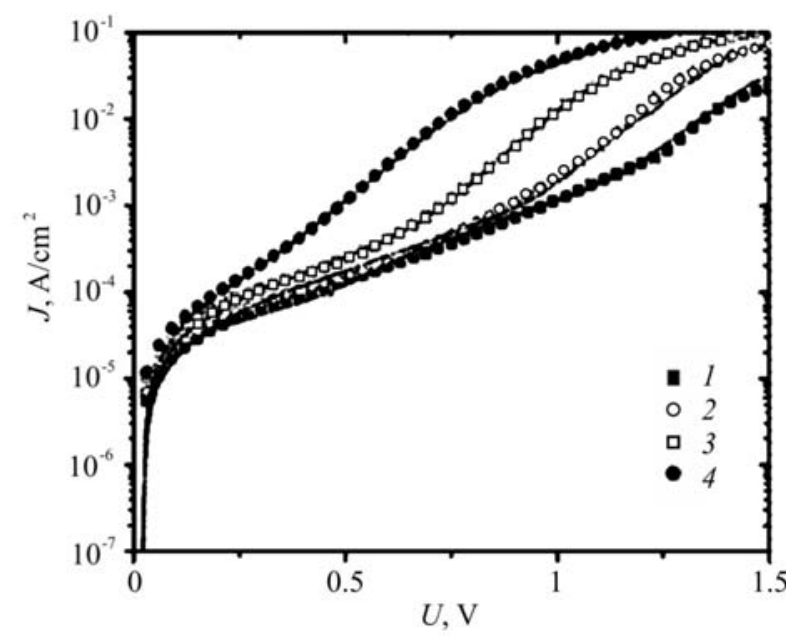

Fig. 4. The effective ideality factor $n$ as a function of the forward bias at different temperatures.

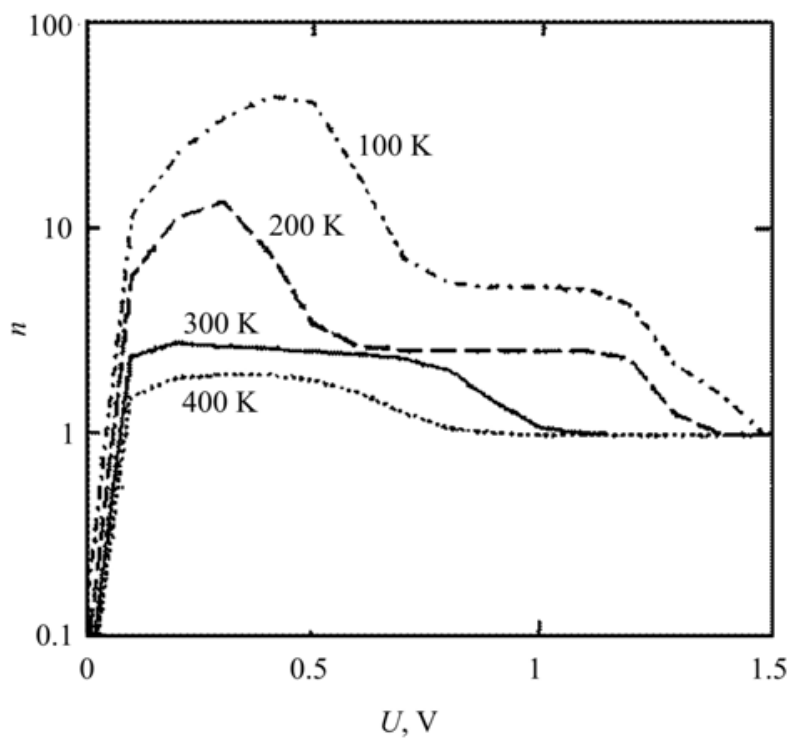

Fig. 5. The experimental forward $I-V$ curves and calculated ones (coordinated with them) for a SC sample made using the capillary LPE technique. The curves were taken at $T=100(1)$, 200 (2), 300 (3) and $400 \mathrm{~K}(4)$.

applied bias, which were obtained according to the following expression:

$$
n=\left(0.0259 \frac{T}{300}\right)^{-1} \lim _{R_{s} \rightarrow 0}\left(J(V, T) / \frac{d}{d V} J(V, T)\right) .
$$

Shown in Fig. 4 are the ideality factor values calculated from Eq. (2) for $I-V$ curves presented in Fig. 3.

Shown in Fig. 5 are the experimental $I-V$ curves and calculated ones (coordinated with them) for a SC sample made by the capillary LPE technique. In this case, the tunnel component contribution into the total current was dominant even at the room temperature. The fitting values of the characteristic energy $\varepsilon_{T}$ varied from 45 up to $170 \mathrm{meV}$ for different samples. The $\lg J(T)$ dependency is linear. This corresponds to the "dislocation shunting" model developed in [5].

The following requirements are to be met lest the tunnel component of the forward current restrict $V_{\mathrm{OC}}$ and $\mathrm{SC}$ photoconversion efficiency. One should decrease, as strongly as possible, the density of growth dislocations. Second, rather high values of the characteristic energy $\varepsilon_{T}$ (that depends on both the effective mass of the tunneling charge carriers and the width of the barrier through which tunneling occurs) promote this.

Based on both our estimations of the tunnel current component and structure investigations of heterojunctions and substrate material, we concluded that the nature of tunnel current in relatively wide $p$ - $n$ junctions (studied by us) is related to the growth dislocations crossing SCR. A fragment of photoconverter structure with disclosed dislocations is shown in Fig. 6.

According to the X-ray diffraction data for textured GaAs samples the level of elastic strains in the layers decreases. This points to partial strain relaxation and appearance of a big number of mismatch dislocations.

Therefore, predominance of the tunnel current component at small biases was observed over the wide temperature range for all the studied SCs made on the textured $n^{+}$-GaAs substrates. In this case, the relief nonuniformities of small curvature radii serve as an additional factor increasing the tunnel current component. However, when preparing the textured GaAs surface, the most disordered semiconductor layers (in particular, those with aggregations of extended defects) are removed in the course of etching as a rule. So, the interfaces become more perfect, and this may result in a decrease of the tunnel current component in relief photoconverters, as compared to their flat analogs. Shown in Fig. 7 are the typical branches of $I-V$ curve for a hetero-photoconverter prototype made on the textured GaAs substrate. The $I-V$ curve was taken in $77 \ldots 400 \mathrm{~K}$ temperature range. One can see from comparison between Figs 1-5 and 7 that the above trend really exists.

\subsection{The effect of thermal treatment on I-V curves in heterostructures grown on flat and textured substrates}

It is known that the solar cells (SCs) may heat up to $T \approx 200{ }^{\circ} \mathrm{C}$ in the course of operation. So we decided to study the effect of thermal treatment (in the $100-200{ }^{\circ} \mathrm{C}$ temperature range for $30 \mathrm{~min}$.) on the mechanisms of current flow in SC.

For SC formed on flat and quasi-grating-like substrates, it was found that the forward branch of $I-V$ curve may be described with two exponents. For SC made on the flat substrate, the predominant current component at $0.1<V<0.4 \mathrm{~V}$ is the tunnel one with the ideality factor $n \approx 3$ and characteristic tunneling energy $\varepsilon_{T} \approx 0.075 \mathrm{eV}$. At $V \geq 0.5 \mathrm{~V}$, the recombination current 


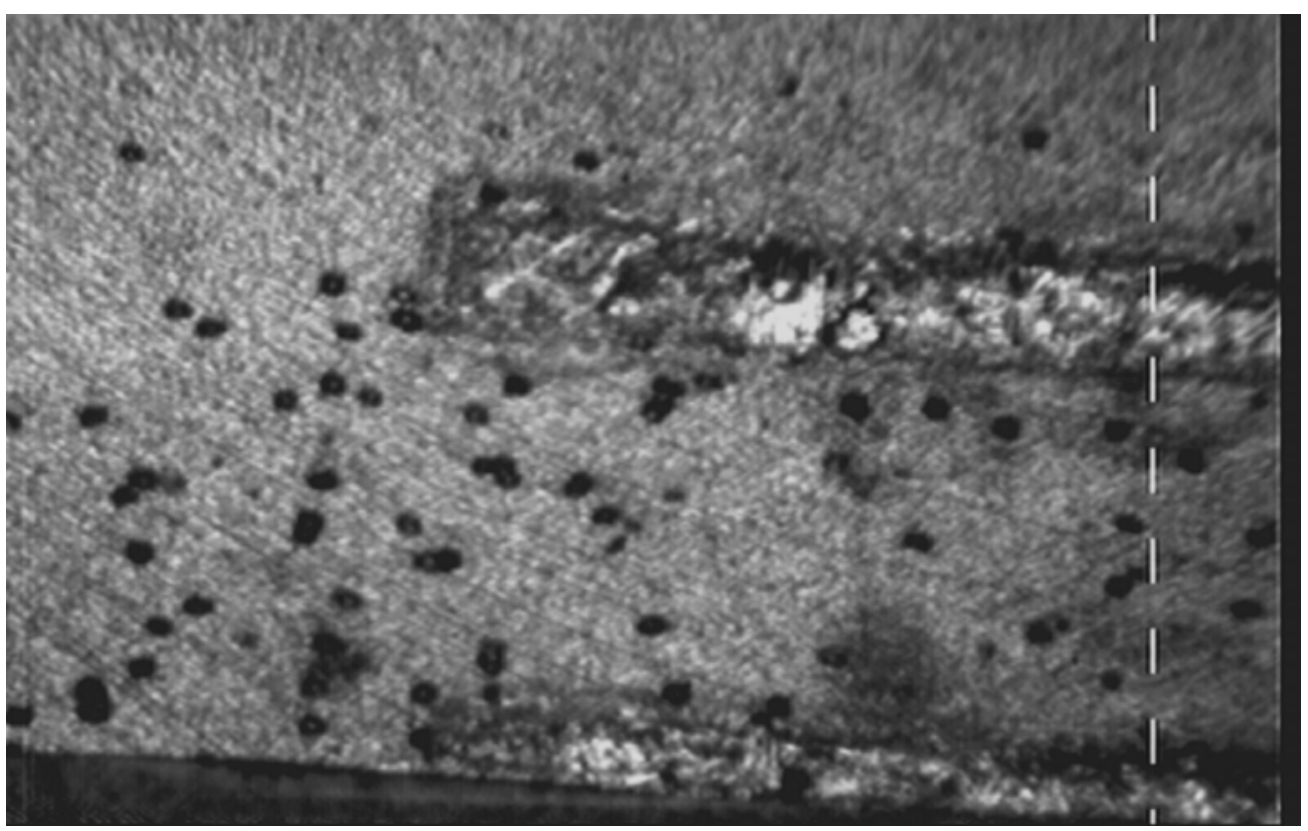

Fig. 6. A fragment of the photoconverter structure with disclosed dislocations.

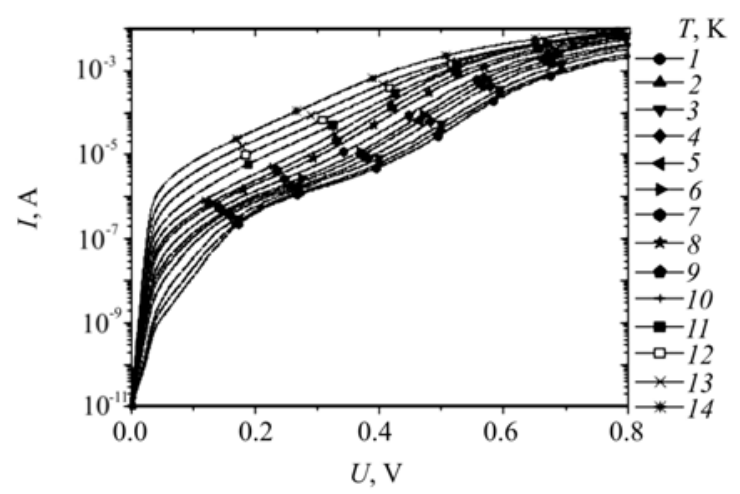

Fig. 7. Forward branches of $I-V$ curves of photoconverter made on the textured GaAs substrate taken at $T=100(1), 120$ (2), 140 (3), 160 (4), 180 (5), 200 (6), 220 (7), 240 (8), 260 (9), 280 (10), 333 (11), 353 (12), 373 (13) and 393 (14) K.

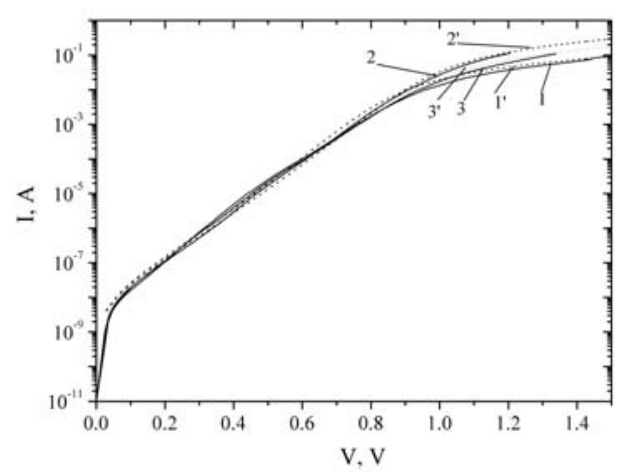

Fig. 8. Forward branch of $I-V$ curve for $\mathrm{SC}$ made on flat substrate: $1\left(1^{\prime}\right)$ - experimental (calculated) $I-V$ curve for the initial sample; $2\left(2^{\prime}\right)$ the same for the sample exposed to thermal treatment $\left(100^{\circ} \mathrm{C}, 30 \mathrm{~min}\right) ; 3\left(3^{\prime}\right)$ - the same for the sample exposed to thermal treatment $\left(200^{\circ} \mathrm{C}, 30 \mathrm{~min}\right)$.

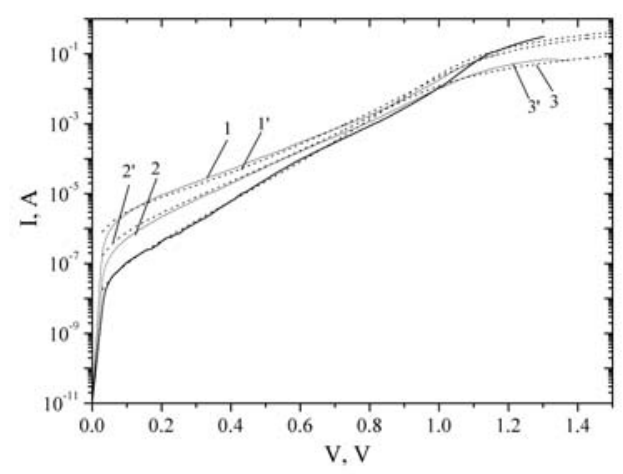

Fig. 9. The forward branch of $I-V$ curve of SC made on the flat substrate: 1, 2, 3 (1', 2', 3') - experimental (calculated) $I-V$ curves (initial and after $\gamma$-irradiation up to doses of $10^{3}$ and $5 \cdot 10^{5} \mathrm{~Gy}$, respectively).

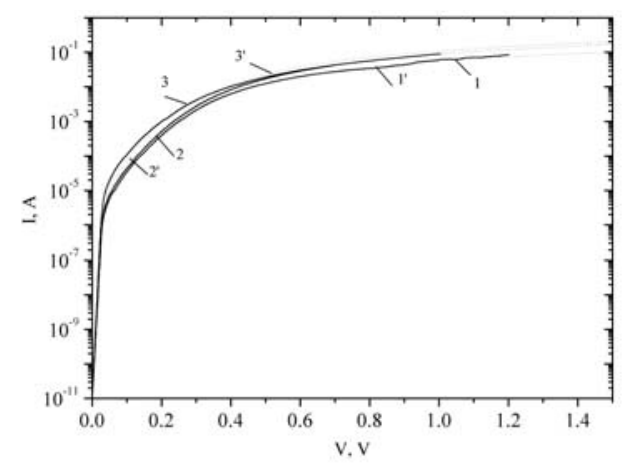

Fig. 10. The forward branch of $I-V$ curve of HPC made on the textured (quasi-grating-like) substrate: 1, 2, 3 (1', 2', 3') experimental (calculated) $I-V$ curves (initial and after $\gamma$ irradiation up to doses of $10^{3}$ and $5 \cdot 10^{5} \mathrm{~Gy}$, respectively). 
component (with $\mathrm{n} \approx 2$ ) dominates (see Fig. 8, curve 1), while the tunneling one is practically insignificant. Thermal treatment at 100 and $200{ }^{\circ} \mathrm{C}$ (Fig. 8, curves 2 and 3 , respectively) does not change both $I-V$ curve slopes and $\varepsilon_{T}$ value. However, as can be seen from Fig. 8, it leads to reduction of the series resistance (from $6 \mathrm{Ohm}$ in the initial sample down to $1.5 \mathrm{Ohm}$ after thermal treatment at $200{ }^{\circ} \mathrm{C}$ for $30 \mathrm{~min}$.). Similar changes are observed for $I-V$ curves of HPC formed on textured substrates, after the thermal treatment at $T=200{ }^{\circ} \mathrm{C}$ for $30 \mathrm{~min}$.

For SC of both types, the reverse current changes insignificantly over the whole range of voltages applied after thermal treatments at $T=100$ and $200{ }^{\circ} \mathrm{C}$. It should be also noted that the experimental forward branches of $I-V$ curves before and after thermal treatments are in a good agreement with the theoretical ones for SC of both types.

\subsection{The effect of ${ }^{60} \mathrm{Co} \gamma$-irradiation on $I-V$ curves of $A l_{x} G a_{1-x} A s-p-G a A s-n-G a A s$ structures}

According to the work plan, we studied the effect of ${ }^{60} \mathrm{Co} \gamma$-irradiation (dose range $10^{3} \ldots .5 \cdot 10^{5} \mathrm{~Gy}$ ) on $I-V$ curves of the $p-\mathrm{Al}_{\mathrm{x}} \mathrm{Ga}_{1-\mathrm{x}} \mathrm{As}-p$-GaAs- $n$-GaAs structures formed on flat and textured substrates. In what follows, we consider the radiation effects in SC at low (up to $\left.10^{3} \mathrm{~Gy}\right)$ doses of $\gamma$-irradiation and in the $5 \cdot 10^{3} \ldots 5 \cdot 10^{5}$ Gy dose range.

The forward branch of $I-V$ curve of initial (before irradiation) SC with flat surfaces is described with two exponents. One can see from Fig. 9 (curve 1) that for the first of them, in 0.01 to $0.7 \mathrm{~V}$ voltage range, the tunnel current with $\varepsilon_{T} \sim 0.1 \mathrm{~V}$ dominates, while for the second one, in $0.7-1 \mathrm{~V}$ voltage range, the recombination current is predominant. The experimental $I-V$ curve of the initial $\mathrm{SC}$ is in agreement with the theoretical one calculated with allowance for the two-exponent model for the current flow (see Fig. 9, curve 1').

$\gamma$-irradiation up to a dose of $10^{3} \mathrm{~Gy}$ leads to an essential change of $I-V$ curve (which now can be described by a single exponent with the slope of $\sim 2$ in 0.01 to $1 \mathrm{~V}$ voltage range). One can see from Fig. 9 that the saturation current decreases by about two orders as compared to that for the initial sample; the calculated $I-V$ curve is in a good agreement with the experimental one. The above effect is not observed at the high $\left(5 \cdot 10^{5} \mathrm{~Gy}\right)$ irradiation dose; in this case, both series resistance and saturation current are increased as compared to those at the irradiation dose of $10^{3} \mathrm{~Gy}$.

In the case of structures with relief (quasi-gratinglike) interfaces, forward branches of $I-V$ curves may be described with a one-exponent model (with the ideality factor of about 2) over the whole range of forward biases. The series resistance of such structures is larger (by the factor of 5-6) than that in the flat structures. If one supposes that the recombination current dominates, then the very high saturation current can be explained with the only assumption: the minority charge carrier lifetime is about $10^{-14} \mathrm{~s}$ (this is unlikely). So, the other assumption seems to be more natural, namely: the tunnel mechanism of current flow involving dislocations is predominant in these structures at the room temperature. Evidently, the concentration of the deep-lying levels that decorate dislocations in these structures is increased. This gives explanation for a rather low $(\sim 0.05 \mathrm{eV})$ characteristic energy of tunneling.

Neither high-, nor low-dose $\gamma$-irradiation serves as a positive factor for the above structures. Low-dose irradiation increases the saturation current; at higher doses it somewhat decreases, but the series resistance grows (see Fig. 10). From the reverse branches of $I-V$ curves for HPC of both types one can see that the reverse current decreases after low-dose irradiation over the whole range of voltages measured. As the irradiation dose is increased up to $5 \cdot 10^{5} \mathrm{~Gy}$, then the reverse current increases slightly.

In the dark current of the barrier structures made on textured $n^{+}$-GaAs substrates, the tunnel current is predominant. This is due to heavy doping of $p^{+}$-emitter and high density of dislocations crossing the $p-n$ junction. Thermal treatment at $T=100-200^{\circ} \mathrm{C}$ affects $I-V$ curves but slightly: the reverse current somewhat decreases, while the series resistance of SC decreases essentially (sometimes by three times). This may indicate at improvement of ohmic contacts.

${ }^{60} \mathrm{Co} \gamma$-irradiation at low doses $\left(D \leq 5 \cdot 10^{5} \mathrm{~Gy}\right)$ somewhat decreases the reverse current; the physicochemical nature of this effect still needs further investigation.

\section{Conclusion}

It was found that the main mechanism of current flow in the forward-biased $p-n$ junctions made on microrelief substrates over a wide temperature range is multistep tunneling of charge carriers along dislocation line (the "dislocation shunt" model). This was confirmed by agreement between the calculated and experimental $I-V$ curves.

\section{Acknowledgement}

This work was supported by the Science and Technology Center in Ukraine (Grant UZB-56(J)).

\section{References}

1. O.Yu. Borkovskaya, N.L. Dmitruk, V.G. Lyapin, and A.V. Sachenko, Thin Solid Films 451/452, p. 402 (2004).

2. N.L. Dmitruk, O.Yu. Borkovskaya, I.B. Mamontova, and I.N. Dmitruk, Sol. Energy Mater. Sol. Cells 76, p. 625 (2003). 
3. V.M. Andreev, in: Y.M. Marshall and D. DimovaMalinovska (Eds.), Photovoltaic and Photoactive Materials-Properties, Technology and Application 80, p.131, Kluwer Acad. Publ., London (2002).

4. V.V. Evstropov, Yu.V. Zhilyaev, M. Jumaeva, and N. Nazarov, Fiz. Tekhn. Poluprov. 31, p. 152 (1997).
5. V.V. Evstropov, M. Jumaeva, Yu.V. Zhilyaev, N. Nazarov, A.A. Sitnikova, and L.M. Fedorov, Fiz. Tekhn. Poluprov. 34, p. 1357 (2000).

6. M.E. Raikh and I.I. Ruzin, Fiz. Tekhn. Poluprov. 19, p. 1217 (1985). 